\title{
Psychometric properties of Persian Version Dispositional Flow Scale -2 (DFS-2)
}

\author{
Hadi Nojavan Alanagh ${ }^{1}$, Hassan Gharayagh Zandi ${ }^{2}$, Rasool Hemayat Talab ${ }^{3}$ \\ ${ }^{I}$ Sports Psychology post graduate student, at Faculty of Physical Education and Sports Science, Tehran \\ University, Tehran \\ ${ }^{2}$ Professor at Faculty of Physical Education and Sports Science, Tehran University, Tehran \\ ${ }^{3}$ Associate Professor at Faculty of Physical Education and Sport Sciences, Tehran University, Tehran, Iran.
}

\begin{abstract}
The current study was done in order to examine the psychometric properties of Persian Version of Dispositional Flow Scale-2 (DFS-2) among Iranian athletes. The original Dispositional Flow Scale-2 contains 36 items and nine subscales. The Dispositional Flow Scale-2 was distributed among the 1180 Iranian athletes with an average age of 20/4 year from different sports. To determine the internal consistency, temporal stability, the validity of exploratory construct and model confirmation, Cronbach's alpha, intraclass correlation coefficient, Exploratory Factor Analysis, confirmatory factor analysis were used respectively. The results showed that Dispositional Flow Scale-2 with nine factors, explained \% 72/527 of total variance and has acceptable internal consistency (0/86) and temporal stability (0/85). Structure of the Dispositional Flow Scale-2 had acceptable fit with the data and all the indicators of goodness of fit confirmed the model. Overall, it seems the Dispositional Flow Scale-2 (DFS-2) has the required validity and reliability for implementation and Interpretation among Iranian athletes.
\end{abstract}

Keywords: Athletes, confirmatory factor, Flow, Reliability, Validity

\section{Introduction}

One of the main reasons for people to play sports is the positive subjective experiences resulting from these activities. A successful and enjoyable performance (good execution or Winning) can result in a positive experience. The best performance of athletes is in competitions in which they give their optimal performance including Full focus and completely absorption in the task at hand, having a sense of confidence and control, while the body effortlessly and automatically acts (14). Csikszentmihalyi (2002) called this ideal experience flow. Flow is an important conceptual and subjective experience in positive psychology. Having Interest in studying the experience of flow during physical activity in sport and exercise psychology is due to identifying the positive and important aspects of experiences of a developed activity. (19)

Csikszentmihalyi, observing the efforts of artists, notices that their work consists of intense involvement in highlighting various activities, and describes modes such as captivation and similar to selfinfatuation. Investigating artists indicated that they need no external requirements to advance and complete their work, and their relation and pleasure in activities belong to completing painting or sculpture. These experiences wound down as soon as their work was completed. Csikszentmihalyi came to the conclusion that these activities, without the need for any additional bonuses, were conducted independently and on their own. (5) Csikszentmihalyi $(1975,2002)$, in his training seminars and research projects, was to examine the characteristics of joy in work and leisure activities. One of his key findings in various activities was the relation between challenge and skill that would later emerge as one of the main components of the flow theory. (4) As Csikszentmihalyi said most likely only when the subject understands the compatibility between individual skills and situational challenges, flow happens. (5)

Csikszentmihalyi (1990) defined flow as an internal motivation and enjoyable experience. His extensive research showed that when individuals get involved in a challenging, intrinsically motivating, and controllable activity, they experience a distinctive psychological state usually called flow. (5) Asakawa (2004) defined flow as an optimal subjective state in which the person feels cognitive efficacy and deep involvement, and has powerful motivation and also experiences high level of experience. This experience itself is so enjoyable that people even with very high costs and only for doing the activities, engage in activities. In addition to fun existing in the activity and intrinsic motivation to continue it, one of the main aspects of experiencing flow is to soak in activities. (1)

In the field of sports, Jackson (1992) defined flow as a "psychological process that includes a state of complete absorption in activity, and it has this experimental feature that creates an experience with internal rewards and it also has an end in itself." (8) Csikszentmihalyi (1975) defined flow as the positive relation between challenge and skill which is called flow channel. If a person ignores the flow channel, then there are two ways to revive the flow again. The first way is that a person can choose less challenging situations which 
comply with their current skills and stimulate them, and the second way is that people, who are looking for situations where the challenges are high, should improve their skills so that they can return again to flow, and strike challenge-skills balance. Jackson and Csikszentmihalyi proposed this point that in order to achieve flow experience in sports, challenges and skills must be physically, mentally, technically and tactically at the same level. (7)

Flow was studied in sports fields, recreation fields and among the top elite athletes. These studies revealed that especially elite athletes tend to experience flow both during in training and competition. (8)

Furthermore, recent research examined possible links between flow and sports performance. Research results have shown that this condition is associated with high levels of performance and is a very positive experience. According to Privette (1983) flow is a topic that is most discussed in relation to peak performance, so that in a two-way statement it can be said that flow experience relates to peak performance. (Or the other way around) (14) In this regard, Csikszentmihalyi $(1975,1993)$ reported a positive relation between flow and performance (4). It seems, based on research findings and theoretical developments, a causal relationship exists between flow and performance that in this regard affects flow performance, and On the other hand, flow also seems to have an impact on performance. (14) In terms of measuring flow, calculating this condition is very challenging because its nature is complex transient and mental. Csikszentmihalyi (1992) noted that flow is a very complex psychological state, and investigators should not hypostatize flow experiences with equating it with a certain score in measuring flow. According to Csikszentmihalyi, the complexity of flow imposes some problems in measuring it, and any kind of measurement only provides a reflection on this state. (14)

As a result, flow cannot be directly assessed as a mental state, and it requires an introspective method. In order to conduct an assessment on flow, athletes must reflect their flow experience retrospectively, which includes the functions of memory and cognitive restructuring to provide an estimate of the severity of the past event or for a specific period of time. (14)

On the other hand, Brewer et al (1991) noted that the impact of performance result on the assessment of retrospective self-report on psychological states can be compromised by the method of introspection. (3) In addition to the standard measurement of flow, interview techniques can provide greater insight about what the athletes think. In addition to the standardized measurement of flow, interview techniques can provide greater insight into what the athletes think. So a multi-dimensional approach including qualitative techniques that offers more specific information about the flow experiences and avoiding misinterpretation of flow experienced in competitive fields is needed. (14)

Studies in the field of flow have been met with some skepticism in some cases. Some of the reasons for this uncertainty are probably due to difficulty in measuring an experimental, subjective mode, since the measurement of subjective experiences such as flow is complex and unreliable, thus challenging, because subjective experiences are among unstable and unconscious characters of people, and there are difficulties regarding how to define these experiences in productive practical terms. (18)

Csikszentmihalyi (1990) proposed nine dimensions of challenge-skills balance, Action-Awareness Merging, clear goals, Unambiguous Feedback, Concentration on the Task at Hand, Sense of Control, and Loss of Self-Consciousness, Time Transformation, and autotelic ecneirepxe . (4) Although definition of flow has a multi-faceted structure, consisting of nine dimensions, Csikszentmihalyi, in measuring flow, emphasized primarily the balance between challenge and skills. He predicted that when challenges and skills are at the top level of any person, flow happens. Furthermore, it was anticipated that other experimental aspects such as anxiety, apathy and fatigue also occur through the relation of challenge- skills. This approach to measuring challenges and skills is the core of experience sampling method. (ESM: experience sampling method; Csikszentmihalyi and Larson, 1987) Whereby the multi-evaluation assessment of flow experience was recorded, while the ESM has proven to be a popular method to assess flow in everyday life; one of the barriers to the use of ESM method in the fields of physical activity is that researchers are often interested in discrete assessment; no need to say, evaluation is disrupted during activity. (5)

Jackson went on to conduct studies in order to understand and evaluate the flow in physical activities. Jackson initially by using qualitative methods studied how the elite performers understand flow and achieve this condition during performance. Jackson, in continuing touching flow in physical activity and especially understanding of the relation flow and other psychological factors, Self-report tool to assess flow. (10) ${ }^{1}$ Flow State Scale (Jackson and Marsh, 1996) and Dispositional Flow Scale (Jackson, et al, 1998) were designed to evaluate the flow experience on a "special event" and "People tendency to experience flow on physical activity" (10) In preparing the FSS and DFS for physical activities, self-report measurement in a manner that is applicable without dysfunction was important; however, a more important goal was to develop a multi-dimensional assessment that instead of focusing primarily on the challenge-skills balance, reflect all 9 dimensions of flow. Since flow has a multi-dimensional structure, this measurement method of flow, having all aspects of it, provides more thorough evaluation than one-dimensional measurement. $(20,12)$ 
Jackson and Marsh (1996) by taking a multi-dimensional method evaluated 36- statement self -report tool, containing four statements for each dimension. Confirmatory factor analysis showed Fitting satisfactory with the nine-factor model of flow. Statistical evaluations have provided a good support for nine-factor structure provides through estimating the correlation among the factors. Factor loadings were in the range of 0.56 to 0.88 with the average of 0.74 . Despite the existence of a very positive relation between 9 factors, regarding the correlation among the factors, Correlations size ranges from 0.18 to 0.72 (average, $r=0.50$ ), which supports the separation of 9 flow factors. (8)

FSS was prepared in order to assess events recently experienced, so Instructions for respondents are based on this fact. DFS was prepared after RSS, using instructions emphasizing the frequency and repetition of flow experience as to assess individual differences in having a tendency to experience flow. (9)

Csikszentmihalyi stated that it is probable that a particular group of people regardless of position enjoy a better psychological condition to experience flow. The phrase of personal internal intention and determination is applied to the experience tendency of flow personal internal intention and determination is the only factor that is somewhat strange and enigmatic. (15) Jackson et al. $(1998 ; 2001)$ identified those psychological factors that are expected to be in connection with this aspect of flow in order to study the constituents of determination of personal internal intention and determination. (10) In any case, Marsh (1997, 1998), for this tool, created the validity of structure as a multistep process started with the factor structure analysis (the interstitial approach) and then analyzing the patterns of relation between one structure and other structures (the interstitial approach) (18) Moreover, Jackson et al (1998; 2001), in addition to reporting on the analysis results of measuring DFS, the results proved the validity of interstitial structure. In theory, it was expected that some patterns of relation exist between flow and logical psychological structure related to flow that a relation was observed between flow and ability to understand, anxiety and intrinsic motivation. (9) Furthermore, some relations between flow and some dimensions of the "sports self-concept" and "athletes use of psychological skills" have been reported. (10) In both studies, dispositional flow had stronger relation with different psychological structures compared to measuring Flow State. Given that all the other structures were also considered at this dispositional level, this finding was predictable. (10) Moreover, the correlation between flow and functioning, perceived skill, perceived success, grading subjective performance and ending position in total were examined. It was reported that the performance aspects of Flow State are positively correlated with perceived skills, perceived success (9), grading of subjective performance and ending position in total (10). These studies showed that FSS and DFS have structure validity and they were applicable in order to measure the experiences of Flow State and dispositional tendency to experience flow.

Marsh and Jackson (1999) reported complex confirmatory factor analyses of DFS and FSS separately and simultaneously. In general, strong support was shown for Construct validity of the tool measuring flow and dispositional types. Factor loadings range from 0.43 to 0.89 on first-order factors for FSS (mean $=0.78$ ) and from 0.29 to 0.86 for DFS (mean = 0.74). Moreover, regarding the investigation in which flow scales were applied, internal reliability estimates (from 0.72 to 0.91 (mean $=0.85$ ) for FSS, and from 0.70 to 0.88 to (mean $=0.82$ for DFS) were reasonable for both scale. (16) Vlachopoulos et al (2000) measured factor validity for flow among Aerobic athletes and reported reliability of $=0 / 65-0 / 84 \alpha$; However, based on three different models of measuring confirmatory factor analysis (CFA), they concluded that validity of all FSS structural factors for the sample were not tenable, because some of the items in the original scale were statistically and conceptually problematic. (20) As a result, Jackson and Eklund (2002) both revised the tools by replacing the problematic items and proposed a new version of flow scales: DFS-2 and FSS-2 (7)

CFA was detected to measure both items and internal validity in the samples presented good data for the revised version. These two scales are only multi-dimensional scales of flow that the author was aware of their satisfactory psychometric properties and they can be used without causing malfunctions. (7)

Zhao et al. (2012), in a study called "The validity and reliability of the Portuguese version of Dispositional Flow Scale -2 in sports", evaluated Reliability and Validity it with the 1437-person sample who participated in sports (906 women and 531 males). The results revealed internal consistency more than 0.74 for the subscales of Dispositional Flow. Moreover, the indices of fitting, CFI (0.95) NNFI (0.95) and RMSEA (0.04) confirmed the validity of the Portuguese version of genital scale of flow. (11)

Kawabata et al. (2008), in a study called "the Flow State scale -2 and Dispositional Flow Scale -2: investigating the factor validity and reliability for Japanese adults", assessed the Reliability and Validity of the scale of Dispositional Flow Scale -2 for 1223- person of the undergraduate students. The research results showed satisfactory amounts for internal consistency $(0.75$ to 0.93$)$ for the subscales of flow. The indices of fitting CFI (0.95) and RMSEA (0.04) supported the factor structure of the Dispositional Flow Scale -2. (13) In order to study flow, it is possible to consider it in a 9-dimension form which was used by Csikszentmihalyi $(1975,1988)$ in flow. Flow experience has been widely supported as a nine-dimensional structure by studies. (7) These characteristics of flow have been clearly carried out in sports by DFS-2 and FSS-2. Using the same 
measuring of flow provides extremely proper condition to assess flow in specific functional positions and it also lets us compare flow throughout all events. (13)

Csikszentmihalyi (1998) had a discussion about the comprehensiveness of flow; however, most of empirical studies have been done on whites. Systematic studies are required in order to examine the intercultural differences and similarities in flow experience. (17) In response to this need, recently three studies have been conducted using the ESM. Two key findings of these studies were: a) flow experience seems inclusive. B) It has cultural differences and depends on conditions and activities by which flow is experienced. (13)

Given these findings, development of studies in the field of flow is essential among non-Western cultures and also in minorities in Western cultures. (17) Duda \& Hayashi (1998) said that studies on the psychological aspects of behavior in sports and exercise which are limited to key groups, such as studies which are running, are contrary to the principles of scientific research. Moreover, they argued that the lack of comparative research in the psychology of sports and exercise leads to Theoretical frameworks being directed towards special and even misleading direction in generalizing assumptions, because not only are FSS-2 and DFS-2 multidimensional scales having proper measurement properties for measuring flow experience in physical activities fields, So the review of generalizing these scales to non-Western cultures has special importance; therefore, the studies that have applied measures of flow must be appropriate according to intercultural perspective, and help to understand the optimal experience in physical activities. (6)

In this regard, despite the abilities of this questionnaire to help sports psychologists, coaches and athletes to improve sports performance and skills, Validity and reliability of these instruments have not been evaluated in Iran. So this study tries to provide useful and efficient tool for sports psychologists and coaches of our country, which should be consistent with Iranian culture. In addition, it wants to answer this question that whether Dispositional Flow Scale -2 is a valid and reliable among Iranian athletes.

\section{Research method}

In terms of aim, this study is an applied one and in terms of collecting data is a descriptive survey.

\section{Statistical population and sample}

The statistical population of this study consists of elite female and male Iranian athletes in 2014 and 2015 in the city of Tehran. Due to lack of accurate and actual size of population, sample selection was conducted based on the purpose of the research. In The first phase, in order to assess the factor reliability of sample size in proportion to the aim of research, 540 subject's male (238) and women (194) were selected with an average age of 19.97. In the second phase, which was conducted in order to determine the confirmatory factor validity and internal consistency, a sample of 540 subject's male (295) and female (245) with an average age of 20.83 were selected. Participants in this two-stage sampling were chosen by available sampling in the different sports and clubs off the country in the elite and non-elite levels.

In the third phase, in order to study the reliability of the 100 participants (64 elites, 36 non-elite) The questionnaires were distributed through available on-site sampling in two phases at an interval of 4 weeks (Kawabata et al., 2004) and then were collected. In three phases, a total of 1180 people (656 men and 524 women), 732 elites and 448 non-elites, participated in the study.

In this research, by an elite athlete we mean an athlete who has a history of being a member of the national team or a winning a rank in a national tournament. Moreover, athletes who participated in the first and second divisions, but did not have a history on national team, and also had not won a rank in a national tournament, were considered elite.

\section{Tools and methods of gathering information}

Dispositional Flow Scale -2 has been designed to evaluate the dispositional side of flow experience. This scale evaluates the general tendency of respondents toward experiencing flow features in a certain environment. The Respondent thinks directly about the overall experience of flow in a particular activity. Dispositional Flow Scale -2 has 9 subscales, each of which consists of 4 items. In order to grade DFS-2, 5-point Likert scale, ranging from 1 (never) to 5 (always), has been used. This scale was designed by Jackson and EKLUND in 2002, and has acceptable validity $(\mathrm{CFI}=0.95, \mathrm{NNFI}=0.93, \mathrm{RMSEA}=0)$ and reliability $(0.80$ to 0.89 ).

\section{The procedure}

First, the questionnaire was translated into Persian by three independent professor of sports psychology. And then the translated text was translated to English by people Fluent in Professional Translation Specialized English texts. Finally, by comparing the two texts in English (original text and text derived from Persian to English translation), the required reforms were carried out and the final Persian version of Dispositional Flow Scale was prepared. Final confirmation of Persian text of the questionnaire and checking the accuracy and logical validity it was conducted by 10 specialists and translators, and required reforms were done. 
In the next stage, the questionnaire was distributed among a small sample (30 male and female athletes) and it was approved in terms of being understandable, and the required modification was done again.

In the next phase the necessary permits were received from the relevant authorities, in order to distribute the questionnaires and after the competition of the competition by the researcher, the questionnaire, along with the necessary explanations among athletes, to evaluate exploratory and confirmatory factor Ratings, was distributed, filled out and collected. At the end, 100 athletes on two separate occasions at an interval of 4 weeks, in order to assess the time reliability of Dispositional Flow Scale -2, completed the questionnaires. The collected questionnaires were graded according to the method of scoring and the scores of each individual was determined in different parts and entered into the computer to be analyzed.

\section{Statistical methods}

Statistical methods used in this study were descriptive and inferential statistics. Descriptive statistics were applied to calculate central scales, dispersion and drawing the diagrams. Then, in order to analyze the data, Kaiser- Meyer- Olkin test was used to determine the appropriateness of the data, Bartlett's Sphericity test was used to assess the factor ability of the data. Then, exploratory factor analysis was applied in order to determine the number of factors and confirmatory factor analysis was used to test the validity of the scale by absolute, comparative and thrifty indicators. Moreover, in order to measure the time reliability, intraclass correlation coefficient was used and Cronbach's alpha coefficient was used to calculate internal consistency of each subscale questions.

\section{The research Results and findings}

In order to evaluate the properties of Dispositional Flow Scale -2, the two following activities took place among athletes: a) determining the validity, b) determining the reliability

In order to determine the validity of this scale, the Factor structure validity method was used. In determining the validity of Factor structure validity, two exploratory and confirmatory factor analyses were applied.

Kaiser- Meyer- Olkin test proved the appropriateness of the data (0.92) and Bartlett's sphericity test confirmed the factor capability of the data $\left(\mathrm{x}^{2}=13939 / 293, \mathrm{df}=630, \mathrm{p}=0.001\right)$

The results of exploratory factor analysis with varimax rotation showed that 9 factors with special amount greater than one were found that determined a total of $72.527 \%$ of the whole questions variance. To check the confirmatory validity, first order confirmatory factor analysis was used that the results indicate the acceptability of absolute fit index $\left(\mathrm{x}^{2} / \mathrm{df}=2.324, \mathrm{GFI}=0.93\right.$ ), comparative ( $\left.\mathrm{CFI}=0.96, \mathrm{NFI}=0.95\right)$, and thrift (RMSEA=0.06). In the following, second-order confirmatory factor analysis was used.

The fit indices of Second order confirmatory factor model in Table 1 indicate that comparative, thrifty and absolute indicators are in acceptable range. In connection with the fit indicators, acceptable fit values of the data were showed by the model; however, since the chi-square statistic is dependent on sample size, the obtained numbers usually become significant. For this reason, usually the observed ratio of the chi-square to degrees of freedom is used as an indicator for the fitting of the data with the model.

Although a well-established rule does not exist in this context, the ratio of three or less than that indicates an acceptable fitting of the data with the model (12). As it can be seen in Table 1, the ratio of chisquare to degrees of freedom was less than 3. In order to calculate the reliability of Dispositional Flow Scale -2, retesting and internal consistency were used. Intraclass correlation was obtained between 0.62 and 0.90 for the items. In connection with the internal consistency of each subscale, Cronbach's alpha test results showed the reliability coefficient of challenge-skill subscale 0.81 , action-awareness merging 0.95 , clear goals 0.84 , unambiguous feedback 0.84 , focus on the task at hand 0.80 , sense of control 0.86 , loss of consciousness 0.91 , time change 0.88 , and experience of internal intention and determination 0.83 , which indicate proper internal consistency of each subscale. (Table 2)

Table 2 Results shows intraclass correlation (ICC) for determining the time reliability of Dispositional Flow Scale -2. As it can the total reliability of the scale is 850 , which is at an acceptable level. Moreover, the time reliability of subscales of balance between challenge and skill, clear goals, unambiguous feedback, focus on the task at hand, a sense of control, loss of consciousness, transformation and change of time, and experiencing the internal intention and determination are $0.69,0.72,0.85,0.81,0.87$ and 0.90 respectively. So the time reliability of subscales is at an acceptable level. 
Table 1: the values of fit index of the confirmatory factor analysis model of the second-order Dispositional Flow Scale -2

\begin{tabular}{|l|l|l|l|}
\hline indicator & abbreviation & Observed value & Acceptable value \\
\hline Chi-square & \multicolumn{1}{|c|}{$\mathrm{x}^{2}$} & 1618.04 & Dependent on sample size \\
\hline Significance level & $\mathrm{P}$ & 0.001 & Dependent on sample size \\
\hline $\begin{array}{l}\text { The ratio of Chi-square to the } \\
\text { degree of freedom }\end{array}$ & \multicolumn{1}{|c|}{$\mathrm{x}^{2} / \mathrm{d} . \mathrm{f}$} & 2.936 & $<3$ \\
\hline Goodness of fit index & GFI & 0.93 & $>0 / 9$ \\
\hline Adjusted goodness of fit & AGFI & 0.90 & $>0 / 9$ \\
\hline Thrifty normalized fit index & PNFI & 0.84 & $>0 / 5$ \\
\hline root-mean-square error & RMSEA & 0.06 & $<0 / 08$ \\
\hline $\begin{array}{l}\text { Bentler and Bonnet normative } \\
\text { fit index }\end{array}$ & NFI & 0.95 & $>0 / 9$ \\
\hline Not normal fit index & NNFI & 0.97 & $>0 / 9$ \\
\hline Comparative fit index & CFI & 0.96 & $>0 / 9$ \\
\hline relative fit index & RFI & 0.90 & $>0 / 9$ \\
\hline Increasing fit index & IFI & 0.94 & $0-1$ \\
\hline
\end{tabular}

Table 2: Results of intraclass correlation coefficient and Cronbach's alpha coefficient for reliability of Dispositional Flow Scale -2

\begin{tabular}{|l|l|l|l|}
\hline subscales & Number of questions & intraclass correlation coefficient & alpha coefficient \\
\hline challenge-skills balance & 4 & 0.69 & 0.81 \\
\hline Action-awareness merging & 4 & 0.62 & 0.95 \\
\hline clear goals & 4 & 0.72 & 0.84 \\
\hline unambiguous feedback & 4 & 0.85 & 0.83 \\
\hline concentration on the task at hand & 4 & 0.90 & 0.80 \\
\hline sense of control & 4 & 0.63 & 0.86 \\
\hline loss of self-consciousness & 4 & 0.81 & 0.91 \\
\hline transformation of time & 4 & 0.87 & 0.88 \\
\hline Autotelic experience & 4 & 0.90 & 0.83 \\
\hline
\end{tabular}

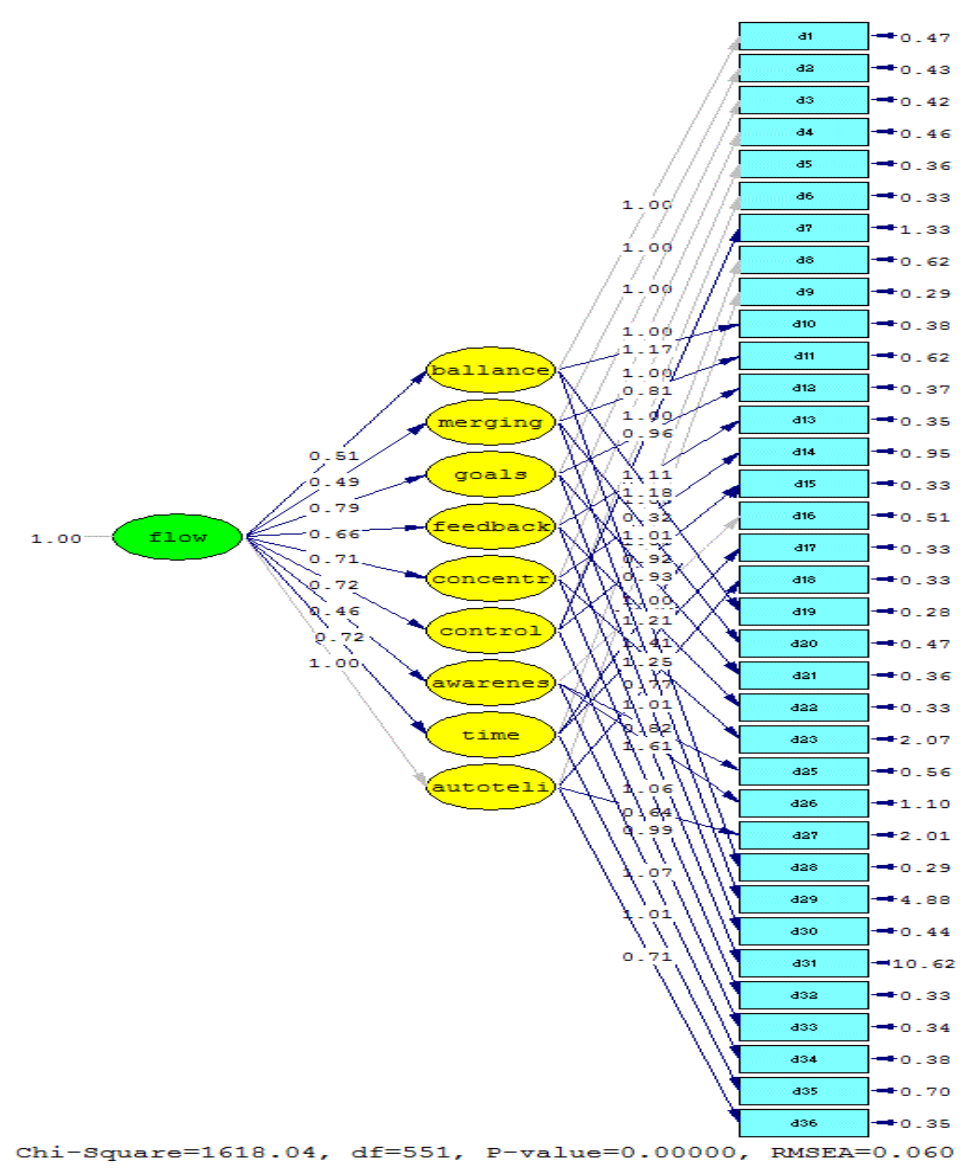

Figure 1 charts of the path of confirmatory factor analysis for Dispositional Flow Scale-2 


\section{Discussion}

The aim of this study was to determine the validity and reliability of Dispositional Flow Scale-2 among Iranian athletes. According to the results of exploratory factor analysis, 36-item scale-2 of flow, with 9 factors defined a total of 72.527 percent of questions variance. In studying question by question, in each subscale no difference was observed between the original and the Persian version, and Questions related to each subscale were placed in their defined positions. In the second stage, in order to confirm the model discovered by exploratory factor analysis, confirmatory factor analysis was applied, whose results were based on fitting indicators and factor loadings indicated 9 factors. Each of the nine factors gained acceptable internal consistency by Cronbach's alpha coefficient method. These coefficients include challenge-skills balance 0.81 , actionawareness merging 0.95 , clear goals 0.84 , unambiguous feedback 0.83 , concentration on the task at hand 0.80 , sense of control 0.86, loss of self-consciousness, 0.91 , transformation of time 0.88 , Autotelic experience 0.83 . The values obtained for internal consistency of Dispositional Flow Scale -2 were higher than those of studies conducted by Joäo Gouveia et al (2012) and Kawabata et al (2008). It seems that this difference is related to the skill level of participants, because the subjects of the study were elite and non-elite athletes participating in national competitions, while Joäo Gouveia et al (2012) and Kawabata et al (2008) studied average and low levels athletes. The internal consistency of the Persian Version was in line and consistent with the results of Jackson et al (2002). The results obtained by intraclass correlation coefficient in investigating the time reliability of the scale showed that the scale and its subscales have high and proper reliability, So that total time reliability of the scale (0.875), challenge-skill balance (0.699), action-awareness merging (0.626), clear goals $(0.854)$, unambiguous feedback (0.854), concentration on the task at hand (0.909), sense of control (0.637), loss of selfconsciousness (0.815), transformation of time (0.874), Autotelic experience (0.905). Intraclass correlation coefficient for total scale and subscales scale was higher than the minimum acceptable level (0.6). These results confirmed Dispositional Flow Scale -2. Results obtained in this study have negligible difference with the values obtained in the study conducted by Kawabata et al (2008). It seems that this consistency is because of the duration (4 weeks) for test-retest the same number of samples.

In general, the indicators of this research were similar to the original version reported by Jackson and Eklund (2002) that was validated with 423 athletes, and confirmed validity and reliability of Dispositional Flow Scale -2. Furthermore, the results of this study were consistent with the findings of Joäo Gouveia et al. (2012) and Kawabata et al (2008) and confirmed their findings.

In summary, this study confirmed the validity and reliability of the Persian version of Dispositional Flow Scale -2, whose original version was designed by Jackson and Eklund (2002) in Australia; Moreover, the results reflected sufficient and satisfactory reliability, and through exploratory and confirmatory factor analysis, confirmed construct validity of this among the Iranian athletes. As a result, it seems possible to use this scale to assess the severity and frequency of flow in sports fields. Due to the fact that flow can help to improve performance, using this scale is recommended to help improve athletic performance.

\section{References}

[1]. Asakawa, K. (2004). Flow experience and autotelic personality in Japanese college students: How do they experience challenges in daily life? Journal of Happiness Studies, 5, 123-154.

[2]. Bassi, M., \& Delle Fave, A. (2004). Adolescence and the changing context of optimal experience in time: Italy 1986-2000. Journal of Happiness Studies, 5, 155-179.

[3]. Brewer, B. W., Van Raalte, J. L., Linder, D. E., \& Van Raalte, N. S. (1991). Peak performance and the perils of retrospective

[4]. Csikszentmihalyi, M. (2000). Beyond boredom and anxiety (25th anniversary Ed.). San Francisco: Jossey-Bass [Original work published in 1975].

[5]. Csikszentmihalyi, M., \& Csikszentmihalyi, I. S. (1988). Introduction to Part IV. In M Csikszentmihalyi \& I. S. Csikszentmihalyi (Eds.), Optimal experience: Psychological studies of flow in consciousness (pp. 251-165). New York, NY: Cambridge University Press.

[6]. Duda, J. L., \& Hayashi, C. T. (1998). Measurement issues in cross-cultural research within sport and exercise psychology. In J. L. Duda (Ed.), Advances in sport and exercise psychology measurement (pp. 471-483). Morgantown, WV: Fitness Information Technology.

[7]. Jackson, S. A., \& Eklund, R. C. (2002). Assessing flow in physical activity: The flow state scale-2 and dispositional flow scale-2. Journal of Sport \& Exercise Psychology, 24, 133-150.

[8]. Jackson, S. A., \& Marsh, H. W. (1996). Development and validation of a scale to measure optimal experience: The Flow State Scale. Journal of Sport \& Exercise Psychology, 18, 17-35.

[9]. Jackson, S. A., Kimiecik, J. C., Ford, S. K., \& Marsh, H. W. (1998). Psychological correlates of flow in sport. Journal of Sport \& Exercise Psychology, 20, 358-378.

[10]. Jackson, S.A., Thomas, PR., Marsh, H.W, \& Smethurst, C.J. (2001) Relationships between flow, self-concept, psychological skills, and performance. Journal of Applied Sport Psychology, 13, 154-178.

[11]. Joäo Gouveia, M., Luís Pais-Ribeiro, J., Moreira Marques, M., \& Carvalho, C. M. (2012). Validity and Reliability of the Portuguese Version of the Dispositional Flow Scale-2 in Exercise. VALIDAD Y FIABILIDAD DE LA Version PORTUGUESA DE LA ESCALA DE FLOW DISPOSICIONAL-2 EN EJERCICIO., 21(1), 81-88.

[12]. Jorskog, K. G. (1969). A general approach to confirmatory maximum likelihood factor analysis .Psychometrical, 34 (9), $183-202$. 
[13]. Kawabata, M., Mallett, C. J., \& Jackson, S. A. (2008). The flow state scale-2 and dispositional flow scale-2: Examination of factorial validity and reliability for Japanese adults. Psychology of Sport and Exercise, 9(4), 465-485.

[14]. Kawabata, M., \& Harimoto, F. (2000). Evaluation of the Japanese version of the Flow State Scale: Analyzing with confirmatory factor analyses. In Proceedings of the 27th annual meeting of the Japanese Society of Sport Psychology, (pp. 8-9). Sapporo, Japan: Japanese Society of Sport Psychology.

[15]. Logan, R. (1988). Flow in solitary ordeals. In M. Csikszentmihalyi \& I. Csikszentmihalyi (Eds.), optimal experience: Psychological studies of flow in consciousness (pp. 172-180). Cambridge: Cambridge University Press.

[16]. Marsh, H. W., \& Jackson, S. A. (1999). Flow experiences in sport: Construct validation of multidimensional, hierarchical state and trait responses. Structural Equation Modeling, 6,343-371.

[17]. Moneta, G. B. (2004). The flow model of intrinsic motivation in Chinese: Cultural and personal moderators. Journal of Happiness Studies, 5, 181-217.

[18]. Nakamura, J., \& Csikszentmihalyi, M. (2002). The concept of flow. In C. R. Snyder, \& S. J. Lopez (Eds.), Handbook of positive psychology (pp. 89-105). New York: Oxford University Press.

[19]. Seligman, M. E. P., \& Csikszentmihalyi, M. (2000). Positive psychology: An introduction. American Psychologist, 55, 5-14.

[20]. Vlachopoulos, S.P., Karageorghis, C.I., \& Terry, PC. (2000). Hierarchical confirmatory factor analysis of the Flow State Scale in exercise. Journal of Sports Sciences, 18, 815-823. 